OPEN ACCESS

Edited by:

Jun Deng, Yale University, United States

Reviewed by: Wenyin Shi,

Thomas Jefferson University, United States

Marianne Aznar, University of Manchester, United Kingdom

*Correspondence: Jianrong $D a$ dai_jianrong@163.com; Junlin $Y_{i}$ yijunlin1969@163.com

Specialty section:

This article was submitted to Radiation Oncology, a section of the journal Frontiers in Oncology

Received: 25 August 2017 Accepted: 05 December 2017 Published: 20 December 2017

Citation: Men $K$, Chen X, Zhang Y, Zhang T, Dai J, Yi J and Li Y (2017) Deep Deconvolutional Neural Network for Target Segmentation of Nasopharyngeal Cancer in Planning Computed Tomography Images.

Front. Oncol. 7:315. doi: 10.3389/fonc.2017.00315

\section{Deep Deconvolutional Neural Network for Target Segmentation of Nasopharyngeal Cancer in Planning Computed Tomography Images}

\author{
Kuo Men, Xinyuan Chen, Ye Zhang, Tao Zhang, Jianrong Dai*, Junlin Yi* and Yexiong Li
}

National Cancer Center/Cancer Hospital, Chinese Academy of Medical Sciences and Peking Union Medical College, Beijing, China

Background: Radiotherapy is one of the main treatment methods for nasopharyngeal carcinoma (NPC). It requires exact delineation of the nasopharynx gross tumor volume (GTVnx), the metastatic lymph node gross tumor volume (GTVnd), the clinical target volume (CTV), and organs at risk in the planning computed tomography images. However, this task is time-consuming and operator dependent. In the present study, we developed an end-to-end deep deconvolutional neural network (DDNN) for segmentation of these targets.

Methods: The proposed DDNN is an end-to-end architecture enabling fast training and testing. It consists of two important components: an encoder network and a decoder network. The encoder network was used to extract the visual features of a medical image and the decoder network was used to recover the original resolution by deploying deconvolution. A total of 230 patients diagnosed with NPC stage I or stage II were included in this study. Data from 184 patients were chosen randomly as a training set to adjust the parameters of DDNN, and the remaining 46 patients were the test set to assess the performance of the model. The Dice similarity coefficient (DSC) was used to quantify the segmentation results of the GTVnx, GTVnd, and CTV. In addition, the performance of DDNN was compared with the VGG-16 model.

Results: The proposed DDNN method outperformed the VGG-16 in all the segmentation. The mean DSC values of DDNN were $80.9 \%$ for GTVnx, $62.3 \%$ for the GTVnd, and 82.6\% for CTV, whereas VGG-16 obtained $72.3,33.7$, and $73.7 \%$ for the DSC values, respectively.

Conclusion: DDNN can be used to segment the GTVnx and CTV accurately. The accuracy for the GTVnd segmentation was relatively low due to the considerable differences in its shape, volume, and location among patients. The accuracy is expected to increase with more training data and combination of MR images. In conclusion, DDNN has the potential to improve the consistency of contouring and streamline radiotherapy workflows, but careful human review and a considerable amount of editing will be required.

Keywords: automatic segmentation, target volume, deep learning, deep deconvolutional neural network, radiotherapy 


\section{INTRODUCTION}

Nasopharyngeal carcinoma (NPC) is a malignant tumor prevalent in southern China. Radiotherapy is one of the main treatments for NPC, and its rapid development has played a significant role in the improvement of tumor control probability. Intensity-modulated radiotherapy and volumetric-modulated radiotherapy (VMAT) have become the state-of-the-art methods for the treatment of NPC over the past two decades $(1,2)$. These technologies can facilitate dose escalation to the tumor target while improving the sparing of organs at risk (OARs), and the dose distribution usually has steep gradients at the target boundary. Modern treatment planning system (TPS) requires exact delineation of the nasopharynx gross tumor volume (GTVnx), the metastatic lymph node gross tumor volume (GTVnd), the clinical target volume (CTV) to be irradiated, and OARs to be spared in planning computed tomography (CT) images so that a radiation delivery plan can be optimized reversely. This task is a type of image segmentation and is usually carried out manually by radiation oncologists based on recommended guidelines (e.g., RTOG 0615 Protocol). However, the manual segmentation (MS) process is time-consuming and operator dependent. It has been reported that the segmentation of a single head-and-neck $(\mathrm{H} \& \mathrm{~N})$ cancer case takes an average of $\sim 2.7 \mathrm{~h}(3)$. This timeconsuming work may be repeated several times during a course of NPC radiotherapy due to a tumor response or significant anatomic changes and alterations. In addition, the accuracy of the segmentation is highly dependent on the knowledge, experience, and preference of the radiation oncologists. Considerable interand intra-observer variation in segmentation of these regions of interest (ROIs) have been noted in a number of studies (4-7).

As a result, a fully automated segmentation method for radiotherapy is helpful to relieve radiation oncologists from the labor-intensive aspects of their work and increase the accuracy, consistency, and reproducibility of ROI delineation. "Atlas-based segmentation" (ABS) (8-10) incorporates a prior knowledge into the process of segmentation and is one of the most widely used and successful image segmentation techniques for biomedical applications. In this type of method, an optimal transformation between the target image to be segmented and a single atlas or multiple atlases containing some ground truth segmentations is computed using deformable registration techniques. Then, all the labeled structures in the atlas image can be propagated through the registration transformation onto the target image automatically. ABS has become a popular method in automatic delineation of target and/or OARs in H\&N radiotherapy (11-17) due to its acceptable results and fully unsupervised mode of operation. Han et al. (11) used the object shape information in the atlas to account

\footnotetext{
Abbreviations: NPC, nasopharyngeal carcinoma; GTVnx, nasopharynx gross tumor volume; GTVnd, metastatic lymph node gross tumor volume; CTV, clinical target volume; OARs, organs at risk; CT, computed tomography; H\&N, head and neck; GT, ground truth; CNNs, convolutional neural networks; DDNN, deep deconvolutional neural network; DSC, Dice similarity coefficient; TCP, tumor control probability; IMRT, intensity-modulated radiotherapy; VMAT, volumetricmodulated radiotherapy; TPS, treatment planning system; ROIs, regions of interest; ABS, Atlas-based segmentation; BN, batch normalized; ReLU, rectified linear non-linearity.
}

for large inter-subject shape differences. Sjöberg et al. (12) applied fusion of multiple atlases to improve the segmentation accuracy than single atlas segmentation. Tao et al. (13) used ABS to reduce interobserver variation and improve dosimetric parameter consistency for OARs. Teguh et al. (14) evaluated autocontouring using ABS and found it was a useful tool for rapid delineation, although editing was inevitable. Sims et al. (15) did a pre-clinical assessment of ABS and showed that it exhibited satisfactory sensitivity; however, careful review and editing were required. Walker et al. (16) concluded ABS was timesaving in generating ROI in $\mathrm{H} \& \mathrm{~N}$, but attending physician approval remained vital. However, there are two main challenges using the ABS method. First, due to the anatomical variations of human organs, it is difficult to build a "universal atlas" for all human organs. The ROI may be considerably different according to the body shape and body size of the patient. The variability should be taken into account to construct a patient-specific atlas from all atlas images, but there are difficulties for target images with a large variability in shape and appearance. Second, a large disadvantage of using ABS is the large computation time that is involved in registering the target image to its atlas image (18). Moreover, it often requires the target image to be aligned to multiple atlases, which will increase the process of registration several times.

Deep learning methods have achieved enormous success in many computer vision tasks, such as image classification (19-21), object detection $(22,23)$, and semantic segmentation (24-26). Convolutional neural networks (CNNs) have become the most popular algorithm for deep learning $(21,27)$. CNNs consist of alternating convolutional and pooling layers to automatically extract multiple-level visual features and have made significant progress in computer-aided diagnosis and automated medical image analysis (28-31). Melendez et al. (29) applied multipleinstance learning for tuberculosis detection using chest X-rays and reported an AUC of 0.86 . Hu et al. (30) proposed a liver segmentation framework based on CNNs and globally optimized surface evolution, yielding a mean Dice similarity coefficient (DSC) of 97\%. Esteva et al. (31) trained a CNN using a large dataset to classify skin cancer and achieved higher accuracy than dermatologists. In addition, CNNs have been applied in the segmentation of many organs and substructures, such as cells (32), nuclei (33), blood vessels (34), neuronal structures (35), brain (36), ventricles (37), liver (38), kidneys (39), pancreas (40), prostate gland (41), bladder (42), colon (43), and vertebrae (44) with relatively better overlap compared with state-of-the-art methods. However, these studies have been confined mostly to the field of radiology.

Furthermore, there has been increasingly more interest in applying CNNs to radiation therapy (45-48). Recently, Ibragimov and Xing (49) used CNNs for OARs segmentation in H\&N CT images and obtained DSC values that varied from $37.4 \%$ for chiasm to $89.5 \%$ for mandible. This was the first report on OAR delineation with CNNs in radiotherapy; however, no target was segmented. In this work, we developed a deep deconvolutional neural network (DDNN) for the segmentation of CTV, GTVnx, and GTVnd for radiotherapy of NPC. The experimental results show that the DDNN can be used to realize the segmentation of NPC targets while planning CT images. DDNN is an end-to-end 
architecture consisting of two important components, including an encoder and a decoder. Different from typical CNNs, we performed a reversed deconvolution at decoder networks to rebuild high-resolution feature maps from low-resolution ones. Our work is the first attempt at applying DDNN for the auto-segmentation of a target for the planning of radiotherapy in NPC.

\section{MATERIALS AND METHODS}

\section{Data Acquisition}

A total of 230 patients diagnosed with NPC stage I or stage II that received radiotherapy during January 2011 to January 2017 in our department were included in our study. All patients were immobilized with a thermoplastic mask (head, neck, shoulder) in the supine position. Simulation contrast CT data were acquired on a Somatom Definition AS 40 (Siemens Healthcare, Forchheim, Germany) or Brilliance CT Big Bore (Philips Healthcare, Best, the Netherlands) system set on helical scan mode with contrast enhancement. CT images were reconstructed using a matrix size of $512 \times 512$ and thickness of $3.0 \mathrm{~mm}$. MR images of all patients were acquired to assist the definition of the targets. Radiation oncologists contoured the GTVnx, the GTVnd, CTV, and OARs in the planning CT using a Pinnacle TPS (Philips Radiation Oncology Systems, Fitchburg, WI, USA) system. The GTVnx was defined as the primary nasopharyngeal tumor mass. The GTVnd was defined as the metastatic lymph nodes. The CTV (CTV1 + CTV2) included GTVnx, GTVnd, high-risk local regions that contain the parapharyngeal spaces, the posterior third of nasal cavities and maxillary sinuses, pterygoid processes, pterygopalatine fossa, the posterior half of the ethmoid sinus, cavernous sinus, base of skull, sphenoid sinus, the anterior half of the clivus, petrous tips, and high-risk lymphatic drainage areas, including bilateral retropharyngeal lymph nodes and level II.

\section{DDNN Model for Segmentation}

In the present study, we introduced a DDNN model to segment the target NPC for radiotherapy. DDNN is an end-to-end segmentation framework that can predict pixel class labels in CT images. Figure 1 depicts the flowchart of the proposed model. As is shown in Figure 2, the DDNN networks consisted of two important components, including an encoder part and a decoder part. The encoder network consisted of 13 convolutional layers

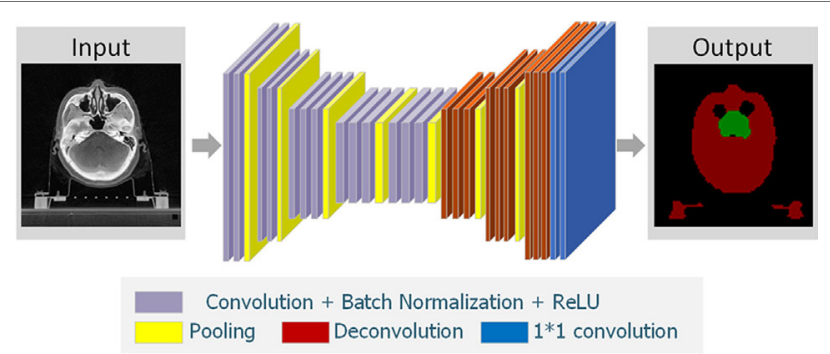

FIGURE 1 | Overall framework of the proposed algorithm. for feature extraction and was used to extract the visual features of the medical image, and the decoder network recovered the original resolution by deploying deconvolution. Specifically, the

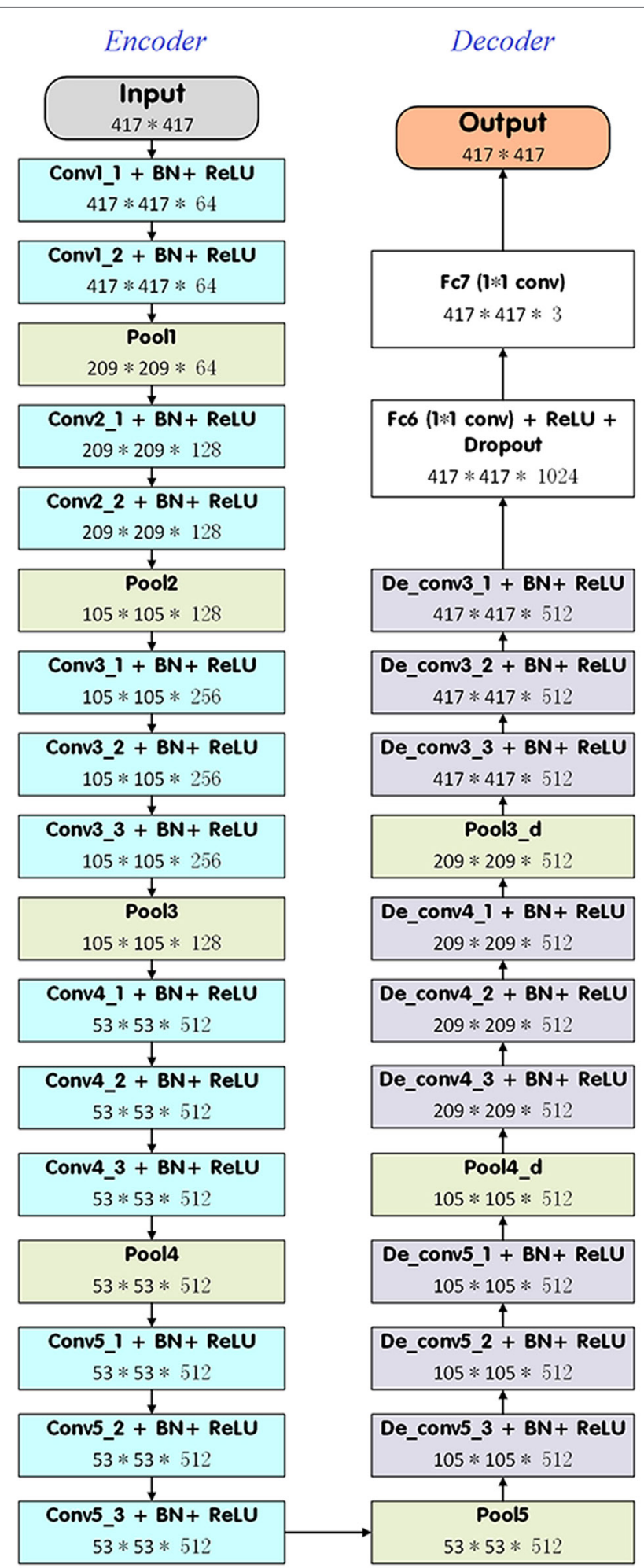

FIGURE 2 | The detailed architecture of deep deconvolutional neural network. 
encoder network layers were based on the VGG-16 architecture (21), used for high-quality image classification. Different from VGG-16, we performed a reversed deconvolution at decoder networks to rebuild high-resolution feature maps from lowresolution. In addition, we replaced the fully connected layers with fully convolutional layers for our segmentation task. With the adaptation, the networks can achieve pixel segmentation in CT images. Please refer to the appendix for more technical specifications of the architecture.

\section{Experiments}

Data from 184 patients out of 230 were chosen randomly as a training set to adjust the parameters of the DDNN model, and the remaining 46 patients were used as the test set to evaluate the performance of the model. In this work, we implemented our model's training, evaluation, error analysis, and visualization pipeline using Caffe (50), which is a popular deep learning framework, and then compiled using cuDNN (51) computational kernels. For the experiments, we adopted data augmentation techniques, such as random cropping and flipping to reduce over fitting. We used stochastic gradient descent with momentum to optimize the loss of function. We set the initial learning rate to 0.0001 , learning rate decay factor to 0.0005 , and decay step size to 2,000 . Instead of using a fixed number of steps, we trained our model until the mean average precision of the training set converged, and then evaluated the model using the validation set. We used NVIDIA TITAN XP GPU for all experiments.

\section{Quantitative Evaluation}

A total of 46 patients were used to assess the performance of the model. MSs were defined as the reference segmentations generated by the experienced radiation oncologists. All the voxels that belong to the MS were extracted and labeled. During the testing phase, all the $2 \mathrm{D}$ CT slices were tested one by one. The input was the 2D CT image, and the final output was pixel-level classification, which was the most likely classification label. Performance of the proposed method was tested and compared with the segmentation of the GTVnx, GTVnd, and CTV. The DSC and the Hausdorff distance $(H)$ were used to quantify the results.

The DSC is defined as shown in Eq. 1 as follows:

$$
\operatorname{DsC}(\mathrm{A}, \mathrm{B})=\frac{2|\mathrm{~A} \cap \mathrm{B}|}{|\mathrm{A}|+|\mathrm{B}|}
$$

where A represents the MS, B denotes the auto-segmented structure and $\mathrm{A} \cap \mathrm{B}$ is the intersection of $\mathrm{A}$ and $\mathrm{B}$. The DSC results in values between 0 and 1 , where 0 represents no intersection at all and 1 reflects perfect overlap of structures $A$ and $B$.

The Hausdorff distance $(H)$ is defined as

$$
H(\mathrm{~A}, \mathrm{~B})=\max (h(\mathrm{~A}, \mathrm{~B}), h(\mathrm{~B}, \mathrm{~A}))
$$

where

$$
h(\mathrm{~A}, \mathrm{~B})=\max \left(\min _{a \in A, b \in B}\|a-b\|\right)
$$

and $\|$.$\| is some underlying norm on the points of A and B.$ As $H(\mathrm{~A}, \mathrm{~B})$ diminishes, the overlap between $\mathrm{A}$ and $\mathrm{B}$ increases.
In addition, the performance of DDNN was compared with VGG-16. The average DSC and Hausdorff distance values for the three targets (GTVnx, GTVnd, and CTV) were analyzed with paired $t$-tests between DDNN and VGG-16. All analyses were performed with a $p$-value set to $<0.05$.

\section{RESULTS}

The results for all tested patients and GTVnx, GTVnd, and CTV values are summarized in Figure 3 and Table 1. The proposed DDNN auto-segmentation showed a better overall agreement than the VGG-16 based auto-segmentation, as shown by the DSC values. The average DSC value of DDNN was $15.4 \%$ higher than the VGG-16 average DSC value $(75.3 \pm 11.3$ vs. $59.9 \pm 22.7 \%$, $p<0.05)$. Automatic delineation with DDNN produced a good result for the GTVnx and CTV, with DSC values of 80.9 and $82.6 \%$, respectively. These values showed a reasonable volume overlap of the auto-segmented contours and the manual contours. The quality of the automatically generated GTVnd was barely satisfactory, with a mean DSC value of $62.3 \%$. The Hausdorff distance values for all targets were reduced by DDNN compared with VGG-16 $(12.6 \pm 11.5$ vs. $23.4 \pm 24.4, p<0.05)$.
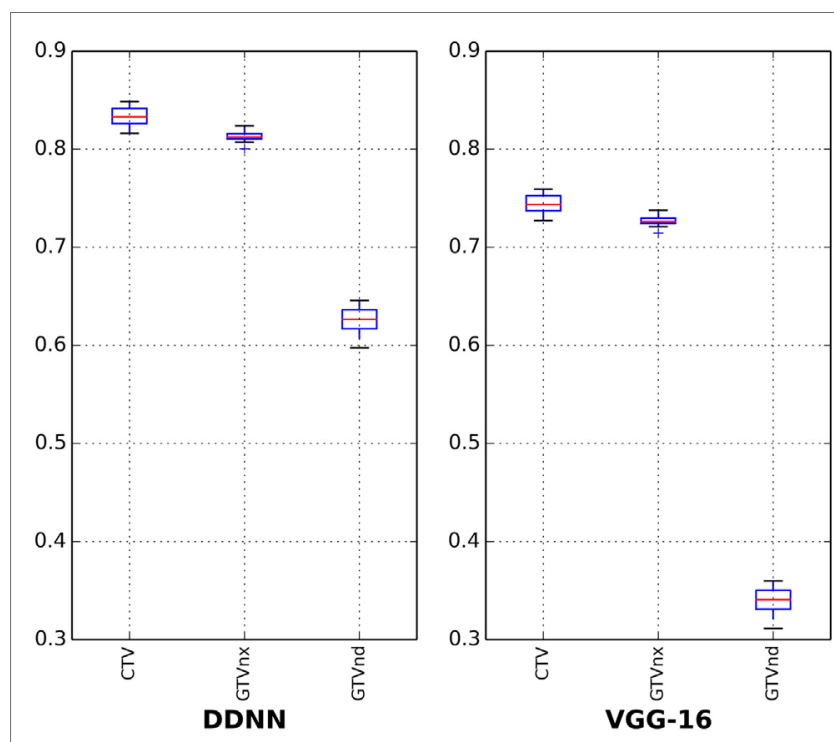

FIGURE 3 | Boxplots obtained from Dice similarity coefficient analyses.

TABLE 1 | Dice similarity coefficient (DSC) and Hausdorff distance for nasopharynx gross tumor volume (GTVnx), metastatic lymph node gross tumor volume (GTVnd), and clinical target volume (CTV).

\begin{tabular}{lccccccc}
\hline & \multicolumn{3}{c}{ DSC (\%) } & & \multicolumn{3}{c}{$\begin{array}{c}\text { Hausdorff distance } \\
\text { (mm) }\end{array}$} \\
\cline { 2 - 4 } \cline { 7 - 8 } Region of interest & CTV & GTVnx & GTVnd & & CTV & GTVnx & GTVnd \\
\hline $\begin{array}{l}\text { Deep deconvolutional } \\
\text { neural network }\end{array}$ & 82.6 & 80.9 & 62.3 & & 6.9 & 5.1 & 25.8 \\
$\begin{array}{l}\text { VGG-16 } \\
\text { VGG }\end{array}$ & 73.7 & 72.3 & 33.7 & & 11.1 & 7.7 & 51.5
\end{tabular}


Figures 4-6 show auto-segmentation of CTV, GTVnx, and GTVnd for test cases, respectively. In these examples, the autosegmented contours of CTV and GTVnx using DDNN were close to the MS contours, although inconsistencies existed. Only a few corrections were necessary to validate the automatic segmentation. However, for the segmentation of the GTVnd, there was some deviation from the MS in shape, volume, and location.

\section{DISCUSSION}

We have designed an automated method to segment CT images of NPC. To the best of our knowledge, this task has not previously

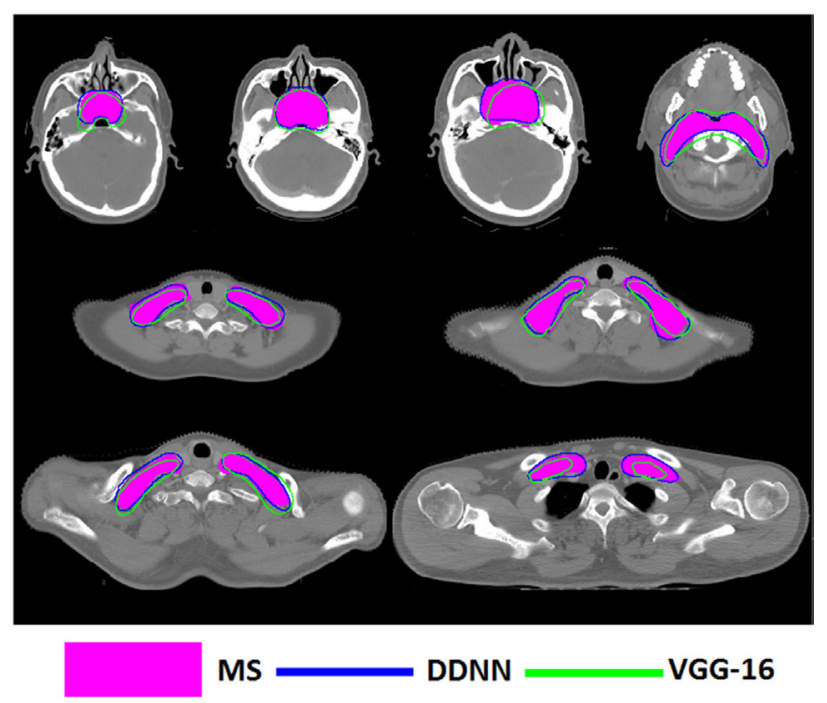

FIGURE 4 | Segmentation results for clinical target volume, shown in transverse computed tomography slices.

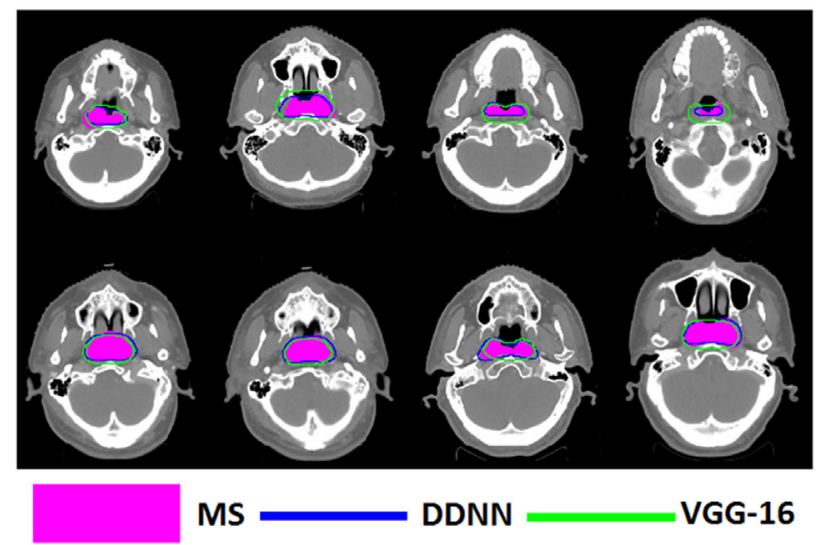

FIGURE 5 | Segmentation results for nasopharynx gross tumor volume, shown in transverse computed tomography slices. been reported. Our results suggest that the proposed DDNN algorithm can learn the semantic information from nasopharyngeal CT data and produce high-quality segmentation of the target. We compared the proposed architecture with the popular Deeplab v2 VGG-16 model. This comparison revealed that our method achieved better segmentation performance. Our DDNN method deployed a deeper encoder and decoder neural network, which used convolutional filters to extract feature and deployed deconvolutional filters to recover the original resolution. Thus, detailed segmented results were learned/predicted better than bilinear interpolation.

Consistency of target delineation is essential for the improvement of radiotherapy outcomes. Leunens et al. (52) demonstrated that inter- and intra-observer variation is considerable. $\mathrm{Lu}$ et al. (53) investigated the interobserver variations in GTV contouring of $\mathrm{H} \& \mathrm{~N}$ patients and reported a DSC value of only $75 \%$. Caravatta et al. (54) evaluated the overlap accuracy of CTV delineation among different radiation oncologists and got a DSC of $68 \%$. Automatic segmentation has the potential to reduce variability of contours among physicians and improve efficiency. The gains in efficiency and consistency are valuable only if accuracy is not compromised. Assessment of accuracy of a segmentation method is complex, because there is no common database or objective volume for comparison. The evaluation of automatic segmentation for radiotherapy planning usually uses the DSC value, thus providing a reasonable basis for comparison. Apparently, our method showed good performance compared with the existing studies regarding the auto-segmentation topic. In addition, such auto-segmentation methods are atlas- and/or model based, and there is no report on segmentation of GTV or CTV using a deep learning method. Regarding the target, the comparison is difficult since $N$-stage (most often N0) and selected levels were quite different from one study to another. For CTV, different previous publications reported mean DSC values of $60 \%$ (55), $60 \%$ (8), 60\% (56), 67\% (14), 77\% (57), $78 \%$ (58), $79 \%$ (59), and $80.2 \%$ (60), whereas the DSC value of DDNN was $82.6 \%$. There are few reports on auto-segmentation of GTVnx or GTVnd. For segmentation of GTVnx, DSC values

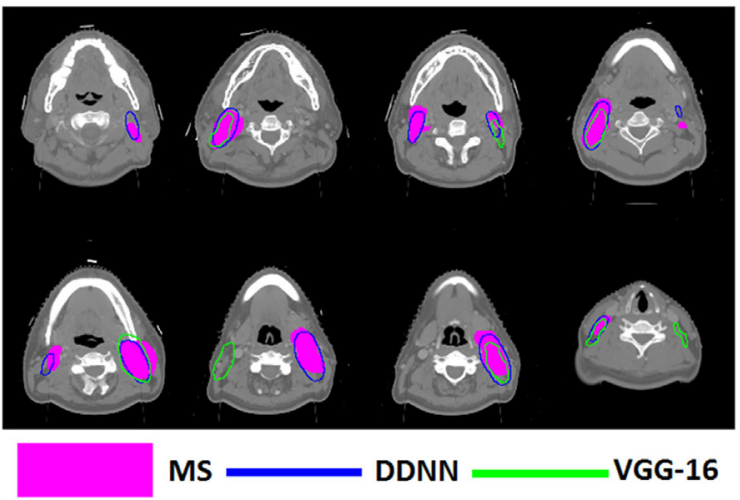

FIGURE 6 | Segmentation results for metastatic lymph node gross tumor volume, shown in transverse computed tomography slices. 
have been reported to be $69.0 \%$ (58) and $75.0 \%$ (61), whereas our proposed method demonstrated a high DSC value of $80.9 \%$. The segmentation of GTVnd reported in the literature has yielded DSC values of $46.0 \%$ (62), and our method showed a DSC value of $62.3 \%$. It is unfair to say our proposed algorithm is superior because the comparison with the published methods was not done with the same dataset; however, it is reasonable to conclude DDNN resulted in good results. Meanwhile, the proposed method learns and predicts in an end-to-end form without post-processing, which makes the inference time of the whole network within seconds.

Although the segmentation accuracy for GTVnd was better than previously reported, it was still too low. There are several reasons for this deficiency. First, this low result was due to lack of soft tissue contrast in CT-based delineation. Second, the GTVnd typically does not have constant image intensity or clear anatomic boundaries, and its shape and location are more variable compared with CTV and GTVnx among different patients. Moreover, there is no GTVnd region in N0 patients, who were also included in our training and test sets. All of these factors will hinder the DDNN model from learning the robust features and making accurate reasoning. Thus, the segmentation accuracy of GTVnd remains unsatisfactory at present. Zijdenbos et al. (63) suggests that a DSC value of $>70 \%$ represents good overlap. Although the segmentation accuracy of CTV and GTVnx exceeded this standard, attending physician oversight remains critical. Imperfect definition of target volumes, which are then used for treatment planning, may result in under dosage of target volumes or an overdose delivered to normal tissues. As a result, the proposed method cannot be applied in an unsupervised fashion in the clinic. Human review and a considerable amount of editing might be required.

There are several limitations to our study. First, a model trained on $\mathrm{NO}$ and $\mathrm{N}+$ patients was used to assess the testing set, including both $\mathrm{NO}$ and $\mathrm{N}+$ patients. This may make the model difficult to converge and reduce the accuracy of the prediction. Second, only one physician delineated the target for each patient but all the patients were delineated by several observers. Although the targets were contoured by experts according to the same guideline for NPC, there was still interobserver variability in all cases. We cannot exclude such possible bias, which challenges the DDNN method. Another limitation of our study is that all of the included patients were stage I or stage II. A target with different stages may have different contrast, shapes, and volumes, thus, influencing the performance of the automated segmentation.

This study mainly focused on NPC target segmentation from CT images. However, MR images in H\&N have superior soft-tissue contrast and the GTV delineation often depends on $\mathrm{MR}$ images. In addition, functional MR may allow accurate location of the tumors. In the future, DDNN is expected to combine with the MR or other types of images to improve target volume delineation. The training set included only 184 patients. Increasing the amount of training data could make the DDNN model more robust, improving the segmentation accuracy. With the initiation of improved target visualization and further improvement of segmentation algorithms in the future, accuracy of auto-segmentation is likely to improve.

\section{CONCLUSION}

Accurate and consistent delineation of tumor target and OARs is particularly important in radiotherapy. Several studies have focused on the segmentation of OARs using deep learning methods. This study shows a method using DDNN architecture to auto-segment nasopharyngeal cancer stage I or stage II in planning CT images. The results suggest that DDNN can be used to segment GTVnx and CTV with high accuracy. The accuracy for GTVnd segmentation was relatively low due to the considerable differences in shape, volume, and location among patients. The performance is expected to improve with multimodality medical images and more training data. In conclusion, DDNN has the potential to improve the consistency of contouring and streamline radiotherapy workflows, but careful human review and a considerable amount of editing will be required.

\section{AVAILABILITY OF DATA AND MATERIALS}

The datasets generated and/or analyzed during the current study are not publicly available due to data security but are available from the corresponding author on reasonable request.

\section{ETHICS STATEMENT}

This study was carried out in accordance with the Declaration of Helsinki and was approved by the Independent Ethics Committee of Cancer Hospital, Chinese Academy of Medical Sciences with the following reference number: NCC2015 YQ-15.

\section{AUTHOR CONTRIBUTIONS}

All authors discussed and conceived of the study design. KM wrote the programs and performed data analysis, and drafted the manuscript. XC and YZ analyzed and interpreted the patients' data. JD, JY, and YL guided the study and participated in discussions and preparation of the manuscript. All authors read, discussed, and approved the final manuscript.

\section{ACKNOWLEDGMENTS}

The authors sincerely thank Dr. Junge Zhang and Dr. Peipei Yang of Institute of Automation, Chinese Academy of Sciences, Dr. Kangwei Liu of LeSee \& Faraday Future AI Institute, and Mr. Rongliang Cheng of Qingdao University of Science and Technology for data mining and editing the manuscript. They also thank the radiation oncologists in our department for the target delineation.

\section{FUNDING}

This work was supported by the National Natural Science Foundation of China (No. 11605291 and No. 11475261), the National Key Projects of Research and Development of China (No. 2016YFC0904600 and No. 2017YFC0107501), and the Beijing Hope Run Special Fund of Cancer Foundation of China (No. LC2015B06). 


\section{REFERENCES}

1. Lee TF, Ting HM, Chao PJ, Fang FM. Dual arc volumetric-modulated arc radiotherapy (VMAT) of nasopharyngeal carcinomas: a simultaneous integrated boost treatment plan comparison with intensity-modulated radiotherapies and single arc VMAT. Clin Oncol (2011) 24(3):196-207. doi:10.1016/j. clon.2011.06.006

2. Moretto F, Rampino M, Munoz F, Ruo Redda MG, Reali A, Balcet V, et al. Conventional 2D (2DRT) and 3D conformal radiotherapy (3DCRT) versus intensity-modulated radiotherapy (IMRT) for nasopharyngeal cancer treatment. Radiol Med (2014) 119(8):634-41. doi:10.1007/s11547-013-0359-7

3. Harari PM, Shiyu S, Wolfgang AT. Emphasizing conformal avoidance versus target definition for IMRT planning in head-and-neck cancer. Int J Radiat Oncol Biol Phys (2010) 77(3):950-8. doi:10.1016/j.ijrobp.2009.09.062

4. Breen S, Publicover J, de Silva S, Pond G, Brock K, O'Sullivan B, et al. Intraobserver and interobserver variability in GTV delineation on FDGPET-CT images of head and neck cancers. Int J Radiat Oncol Biol Phys (2007) 68(3):763-70. doi:10.1016/j.ijrobp.2006.12.039

5. Feng MU, Demiroz C, Vineberg KA, Balter JM, Eisbruch A. Intra-observer variability of organs at risk for head and neck cancer: geometric and dosimetric consequences. Fuel Energy Abstr (2010) 78(3):S444-5. doi:10.1016/j. ijrobp.2010.07.1044

6. Yamazaki H, Hiroya S, Takuji T, Naohiro K. Quantitative assessment of inter-observer variability in target volume delineation on stereotactic radiotherapy treatment for pituitary adenoma and meningioma near optic tract. Radiat Oncol (2011) 6(1):10. doi:10.1186/1748-717X-6-10

7. Vinod SK, Myo M, Michael GJ, Lois $\mathrm{CH}$. A review of interventions to reduce inter-observer variability in volume delineation in radiation oncology. J Med Imaging Radiat Oncol (2016) 60(3):393-406. doi:10.1111/1754-9485. 12462

8. Iglesias JE, Sabuncu MR. Multi-atlas segmentation of biomedical images: a survey. Med Image Anal (2015) 24(1):205-19. doi:10.1016/j.media.2015. 06.012

9. Cabezas M, Oliver A, Lladó X, Freixenet J, Cuadra MB. A review of atlas-based segmentation for magnetic resonance brain images. Comput Methods Programs Biomed (2011) 104(3):e158-77. doi:10.1016/j.cmpb.2011. 07.015

10. Cuadra MB, Duay V, Thiran JP. Atlas-Based Segmentation. Handbook of Biomedical Imaging. USA: Springer (2015). p. 221-44.

11. Han X, Hoogeman MS, Levendag PC, Hibbard LS, Teguh DN, Voet P, et al. Atlas-based auto-segmentation of head and neck CT images. International Conference on Medical Image Computing and Computer-assisted Intervention. Berlin, Heidelberg: Springer (2008). p. 434-41.

12. Sjöberg C, Martin L, Christoffer G, Silvia J, Anders A, Anders M. Clinical evaluation of multi-atlas based segmentation of lymph node regions in head and neck and prostate cancer patients. Radiat Oncol (2013) 8(1):229. doi:10.1186/1748-717X-8-229

13. Tao CJ, Yi JL, Chen NY, Ren W, Cheng J, Tung S, et al. Multi-subject atlasbased auto-segmentation reduces interobserver variation and improves dosimetric parameter consistency for organs at risk in nasopharyngeal carcinoma: a multi-institution clinical study. Radiother Oncol (2015) 115(3):407-11. doi:10.1016/j.radonc.2015.05.012

14. Teguh DN, Levendag PC, Voet PW, Al-Mamgani A, Han X, Wolf TK, et al. Clinical validation of atlas-based auto-segmentation of multiple target volumes and normal tissue (swallowing/mastication) structures in the head and neck. Int J Radiat Oncol Biol Phys (2010) 81(4):950-7. doi:10.1016/j.ijrobp.2010. 07.009

15. Sims R, Isambert A, Grégoire V, Bidault F, Fresco L, Sage J, et al. A pre-clinical assessment of an atlas-based automatic segmentation tool for the head and neck. Radiother Oncol (2009) 93(3):474-8. doi:10.1016/j.radonc.2009. 08.013

16. Walker GV, Awan M, Tao R, Koay EJ, Boehling NS, Grant JD, et al. Prospective randomized double-blind study of atlas-based organ-at-risk autosegmentation-assisted radiation planning in head and neck cancer. Radiother Oncol (2014) 112(3):321-5. doi:10.1016/j.radonc.2014.08.028

17. Raudaschl PF, Zaffino P, Sharp GC, Spadea MF, Chen A, Dawant BM, et al. Evaluation of segmentation methods on head and neck CT: autosegmentation challenge 2015. Med Phys (2017) 44(5):2020-36. doi:10.1002/ mp. 12197
18. Langerak TR, Berendsen FF, Van der Heide UA, Kotte AN, Pluim JP. Multiatlas-based segmentation with preregistration atlas selection. Med Phys (2013) 40(9):091701. doi:10.1118/1.4816654

19. Krizhevsky A, Sutskever I, Hinton GE. Imagenet classification with deep convolutional neural networks. Advances in Neural Information Processing Systems. Nevada, United States (2012). p. 1097-105.

20. Sermanet P, David E, Zhang X, Michael M, Rob F, Yann L. OverFeat: Integrated Recognition, Localization and Detection using Convolutional Networks. (2014) arXiv:1312.6229.

21. Simonyan K, Zisserman A. Very Deep Convolutional Networks for Large-scale Image Recognition. (2014) arXiv:1409.1556.

22. Girshick R, Jeff D, Trevor D, Jitendra M. Rich feature hierarchies for accurate object detection and semantic segmentation. Proceedings of the IEEE Conference on Computer Vision and Pattern Recognition. Columbus, United States (2014). p. 580-7.

23. Ren S, He K, Girshick R, Sun J. Faster R-CNN: towards real-time object detection with region proposal networks. In Advances in Neural Information Processing Systems. Montreal, Canada (2015). p. 91-9.

24. Chen LC, George P, Iasonas K, Kevin M, Alan LY. Semantic Image Segmentation with Deep Convolutional Nets and Fully Connected CRFs. (2014) arXiv:1412.7062.

25. Long J, Shelhamer E, Darrell T. Fully convolutional networks for semantic segmentation. Proceedings of the IEEE Conference on Computer Vision and Pattern Recognition. Boston, United States (2015). p. 3431-40.

26. Zheng S, Sadeep J, Bernardino R, Vibhav V, Su ZZ, Du DL, et al. Conditional random fields as recurrent neural networks. Proceedings of the IEEE International Conference on Computer Vision. Santiago, Chile (2015). p. 1529-37.

27. He K, Zhang X, Ren S, Sun J. Deep residual learning for image recognition. Proceedings of the IEEE Conference on Computer Vision and Pattern Recognition. Las Vegas, United States. (2016). p. 770-8.

28. Crown WH. Potential application of machine learning in health outcomes research and some statistical cautions. Value Health (2015) 18(2):137-40. doi:10.1016/j.jval.2014.12.005

29. Melendez J, Ginneken BV, Maduskar P, Philipsen RH, Reither K, Breuninger M, et al. A novel multiple-instance learning-based approach to computer-aided detection of tuberculosis on chest x-rays. IEEE Trans Med Imaging (2015) 34(1):179-92. doi:10.1109/TMI.2014.2350539

30. Hu P, Wu F, Peng J, Liang P, Kong D. Automatic 3D liver segmentation based on deep learning and globally optimized surface evolution. Phys Med Biol (2016) 61(24):8676. doi:10.1088/1361-6560/61/24/8676

31. Esteva A, Kuprel B, Novoa RA, Ko J, Swetter SM, Blau HM, et al. Dermatologistlevel classification of skin cancer with deep neural networks. Nature (2017) 542(7639):115-8. doi:10.1038/nature21056

32. Song Y, Tan EL, Jiang X, Cheng JZ, Ni D, Chen S, et al. Accurate cervical cell segmentation from overlapping clumps in Pap smear images. IEEE Trans Med Imaging (2017) 36:288-300. doi:10.1109/TMI.2016.2606380

33. Xing F, Xie Y, Yang L. An automatic learning-based framework for robust nucleus segmentation. IEEE Trans Med Imaging (2016) 35(2):550-66. doi:10.1109/TMI.2015.2481436

34. Fu H, Xu Y, Wong DWK, Liu J. Retinal vessel segmentation via deep learning network and fully-connected conditional random fields. Biomedical Imaging (ISBI), 2016 IEEE 13th International Symposium on. IEEE. Prague, Czech Republic (2016). p. 698-701.

35. Drozdzal M, Vorontsov E, Chartrand G, Kadoury S, Pal C. The importance of skip connections in biomedical image segmentation. International Workshop on Large-Scale Annotation of Biomedical Data and Expert Label Synthesis. Athens, Greece (2016). p. 179-87.

36. Kamnitsas K, Ledig C, Newcombe VF, Simpson JP, Kane AD, Menon DK, et al. Efficient multi-scale 3D CNN with fully connected CRF for accurate brain lesion segmentation. Med Image Anal (2017) 36:61. doi:10.1016/j. media.2016.10.004

37. Tran PV. A Fully Convolutional Neural Network for Cardiac Segmentation in Short-Axis MRI. (2016) arXiv:1604.00494.

38. Ben-Cohen A, Diamant I, Klang E, Amitai M, Greenspan H. Fully convolutional network for liver segmentation and lesions detection. International Workshop on Large-Scale Annotation of Biomedical Data and Expert Label Synthesis. Athens, Greece (Vol. 10008) (2016). p. 77-85.

39. Thong W, Kadoury S, Piche N, Pal CJ. Convolutional networks for kidney segmentation in contrast-enhanced CT scans. Computer Methods in 
Biomechanics and Biomedical Engineering: Imaging \& Visualization. Tel Aviv, Israel (2016). p. 1-6.

40. Roth HR, Lu L, Farag A, Sohn A, Summers RM. Spatial aggregation of holistically-nested networks for automated pancreas segmentation. International Conference on Medical Image Computing and Computer-Assisted Intervention. Athens, Greece (2016). p. 451-9.

41. Cheng R, Roth HR, Lu L, Wang S, Turkbey B, Gandler W. Active appearance model and deep learning for more accurate prostate segmentation on MRI. Med Imaging Image Process (2016) 9784:97842I. doi:10.1117/12.2216286

42. Cha KH, Hadjiiski LM, Samala RK, Chan HP, Cohan RH, Caoili EM, et al. Bladder cancer segmentation in CT for treatment response assessment: application of deep-learning convolution neural network - a pilot study. Tomography (2016) 2:421-9. doi:10.18383/j.tom.2016.00184

43. Xu Y, Li Y, Liu M, Wang Y, Lai M, Chang EIC. Gland instance segmentation by deep multichannel side supervision. International Conference on Medical Image Computing and Computer-Assisted Intervention. Athens, Greece (2016). p. 496-504.

44. Korez R, Likar B, Pernus F, Vrtovec T. Model-based segmentation of vertebral bodies from MR images with 3D CNNs. International Conference on Medical Image Computing and Computer-Assisted Intervention. Athens, Greece (2016). p. 433-41.

45. Bibault JE, Giraud P, Burgun A. Big data and machine learning in radiation oncology: state of the art and future prospects. Cancer Lett (2016) 382(1): 110-7. doi:10.1016/j.canlet.2016.05.033

46. Cha KH, Hadjiiski L, Samala RK, Chan HP, Caoili EM, Cohan RH. Urinary bladder segmentation in CT urography using deep-learning convolutional neural network and level sets. Med Phys (2016) 43(4):1882-96. doi:10.1118/1.4944498

47. Hu P, Wu F, Peng J, Bao Y, Feng C, Kong D. Automatic abdominal multi-organ segmentation using deep convolutional neural network and time-implicit level sets. Int J Comput Assist Radiol Surg (2017) 12(3):399-411. doi:10.1007/ s11548-016-1501-5

48. Han X. MR-based synthetic CT generation using a deep convolutional neural network method. Med Phys (2017) 44(4):1408-19. doi:10.1002/mp.12155

49. Ibragimov B, Xing L. Segmentation of organs-at-risks in head and neck CT images using convolutional neural networks. Med Phys (2017) 44(2):547-57. doi: $10.1002 / \mathrm{mp} .12045$

50. Jia Y, Shelhamer E, Donahue J, Karayev S, Long J, Girshick R, et al. Caffe: convolutional architecture for fast feature embedding. ArXiv (1408) 2014:5093.

51. Chetlur S, Woolley C, Vandermersch P, Cohen J, Tran J, Catanzaro B, et al. cuDNN: efficient primitives for deep learning. ArXiv (1410) 2014:0759.

52. Leunens G, Menten J, Weltens C, Verstraete J, Schueren EVD. Quality assessment of medical decision making in radiation oncology: variability in target volume delineation for brain tumours. Radiother Oncol (1993) 29(2):169-75. doi:10.1016/0167-8140(93)90243-2

53. Lu L, Cuttino L, Barani I, Song S, Fatyga M, Murphy M, et al. SU-FF-J-85: inter-observer variation in the planning of head/neck radiotherapy. Med Phys (2006) 33(6):2040. doi:10.1118/1.2240862
54. Caravatta L, Macchia G, Mattiucci GC, Sainato A, Cernusco NL, Mantello G, et al. Inter-observer variability of clinical target volume delineation in radiotherapy treatment of pancreatic cancer: a multi-institutional contouring experience. Radiat Oncol (2014) 9(1):198. doi:10.1186/1748-717X-9-198

55. Chen A, Deeley MA, Niermann KJ, Moretti L, Dawant BM. Combining registration and active shape models for the automatic segmentation of the lymph node regions in head and neck CT images. Med Phys (2010) 37(12):6338-46. doi:10.1118/1.3515459

56. Jean-François D, Andreas B. Atlas-based automatic segmentation of head and neck organs at risk and nodal target volumes: a clinical validation. Radiat Oncol (2013) 8(1):1-11. doi:10.1186/1748-717X-8-154

57. Qazi AA, Pekar V, Kim J, Xie J, Breen SL, Jaffray DA. Auto-segmentation of normal and target structures in head and neck CT images: a feature-driven model-based approach. Med Phys (2011) 38(11):6160-70. doi:10.1118/1.3654160

58. Tsuji SY, Hwang A, Weinberg V, Yom SS, Quivey JM, Xia P. Dosimetric evaluation of automatic segmentation for adaptive IMRT for head-and-neck cancer. Int J Radiat Oncol Biol Phys (2010) 77(3):707-14. doi:10.1016/j. ijrobp.2009.06.012

59. Stapleford LJ, Lawson JD, Perkins C, Edelman S, Davis L, Mcdonald MW, et al. Evaluation of automatic atlas-based lymph node segmentation for head-andneck cancer. Int J Radiat Oncol Biol Phys (2010) 77(3):959-66. doi:10.1016/j. ijrobp.2009.09.023

60. Gorthi S, Duay V, Houhou N, Cuadra MB, Schick U, Becker M. Segmentation of head and neck lymph node regions for radiotherapy planning using active contour-based atlas registration. IEEE J Sel Topics Signal Process (2009) 3(1):135-47. doi:10.1109/JSTSP.2008.2011104

61. Yang J, Beadle BM, Garden AS, Schwartz DL, Aristophanous M. A multimodality segmentation framework for automatic target delineation in head and neck radiotherapy. Med Phys (2015) 42(9):5310-20. doi:10.1118/1.4928485

62. Yang J, Beadle BM, Garden AS, Gunn B, Rosenthal D, Ang K, et al. Autosegmentation of low-risk clinical target volume for head and neck radiation therapy. Pract Radiat Oncol (2014) 4(1):e31-7. doi:10.1016/j.prro.2013. 03.003

63. Zijdenbos AP, Dawant BM, Margolin RA, Palmer AC. Morphometric analysis of white matter lesions in MR images: method and validation. IEEE Trans Med Imaging (1994) 13(4):716-24. doi:10.1109/42.363096

Conflict of Interest Statement: The authors declare that the research was conducted in the absence of any commercial or financial relationships that could be construed as a potential conflict of interest.

Copyright $\odot 2017$ Men, Chen, Zhang, Zhang, Dai, Yi and Li. This is an open-access article distributed under the terms of the Creative Commons Attribution License (CC BY). The use, distribution or reproduction in other forums is permitted, provided the original author(s) or licensor are credited and that the original publication in this journal is cited, in accordance with accepted academic practice. No use, distribution or reproduction is permitted which does not comply with these terms. 


\section{APPENDIX}

\section{Architecture of Deep Deconvolutional Neural Network (DDNN)}

As is shown in Figure 2, the architecture of the proposed DDNN consisted of two parts, each of which had its own role. The encoder networks consisted of 13 convolutional layers for feature extraction. All the kernels of convolutional layers had a window size of $3 \times 3$, a stride of 1 , and a padding of 1 pixel. In addition, there was a batch normalized option following each convolution layer and then an element-wise rectified linear non-linearity $\max (0, x)$ was applied. The pooling options were added after the layers of conv1_2, conv2_2, conv3_3, conv4_3, conv5_3, de_conv5_1, and de_conv4_1 in order to get the robust feature. Specifically, the input size of the medical images in this work was cropped to $417 \times 417$ with 3 channels. Conv1_1 and conv1_2 convolved the input to $417 \times 417 \times 64$, and then reduced to $209 \times 209 \times 64$ feature maps using pooling option with kernel size of $3 \times 3$, a stride of 2 , and a padding of 1 pixel. Similarly, the layers of conv2_1 and conv2_2 took pool1 as input. After using
$3 \times 3$ convolution with a stride of 1 and a padding of 1 pixel, it produced $105 \times 105 \times 256$ feature maps and then was pooled by pool 2 and convolved by conv3, conv4, and conv5. The max pooling options of pool 4 and pool5 with $3 \times 3$ filter size, pad 1 and stride 1 , resulted in a $53 \times 53 \times 512$ output. The pooling options reduced the spatial size of feature map, so the feature map needed to be recovered to the original spatial size for segmentation task. Most previous methods used bilinear interpolation to get high-resolution image; however, a coarse segmentation was not enough to produce good performance for nasopharyngeal cancer. Therefore, the decoder part deployed a deep deconvolution neural network which took pool5 as input and a serial of deconvolution layers for upsampling. All the deconvolutional layers used $3 \times 3$ convolution with the padding size of 1 . At de_conv5_3, de_conv4_3, and de_conv3_3, the stride was set to 2 . For others, the stride was set to 1 . After $8 \times$ enlarging, the feature maps recovered the high resolution as same as input. At fc6 and fc7 layers, we replaced fully connected layer with $1 \times 1$ convolution. Thus, we can carry on the pixel-level classification for the segmentation task. The final outputs generated predicted label for each pixel. 